Recepción: 08 / 11 / 2016

Aceptación: 17 / 01 / 2017

Publicación: 29 / 04 / 2017

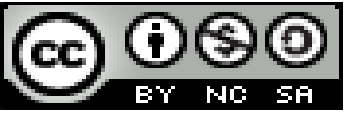

Ciencias económicas y empresariales

Artículo de investigación

\title{
Factores de fracaso y éxito en las empresas familiares. El proceso de sucesión
}

\author{
Factors of failure and success in family businesses. The process of succession
}

Fatores de falha e sucesso em empresas familiares. O processo de sucessão

\author{
Walter I. Navas-Bayona ${ }^{\mathrm{I}}$ \\ winavas@sangregorio.edu.ec \\ Franklin J. González-Soriano II \\ frankgs@hotmail.com \\ Marcelo E. Mendoza-Vinces ${ }^{\text {III }}$ \\ memendoza@sangregorio.edu.ec
}

\section{Correspondencia: winavas@sangregorio.edu.ec}

\footnotetext{
I Magister en Administración de Empresas, Ingeniero Industrial, Docente de la Universidad San Gregorio de Portoviejo, Portoviejo, Ecuador.

II Magister en Gerencia Educativa, Especialista en Gestión de Procesos Educativos, Ingeniero en Sistemas Computacionales, Docente de la Universidad San Gregorio de Portoviejo, Portoviejo, Ecuador.

III Magister en Contabilidad y Auditoría, Contador Público, Ingeniero en Contabilidad y Auditoría, Docente de la Universidad San Gregorio de Portoviejo, Portoviejo, Ecuador.
} 


\section{Resumen}

Las empresas familiares son en el mundo, cada vez más importantes, tanto a nivel económico como social, pues en diversos sectores se han convertido en motores del tejido empresarial. Sin embargo, actualmente enfrentan problemas que en gran medida tributan al proceso de sucesión. Este trabajo tiene como objetivo exponer los problemas que enfrentan hoy las empresas familiares y los elementos para minimizarlos a partir de la literatura publicada sobre este tema.

Palabras clave: Empresas familiares; sucesión en las empresas familiares; éxito de las empresas familiares; problemas de las empresas.

\section{Abstract}

Family businesses are in the world, increasingly important, both economically and socially, because in various sectors have become drivers of the business fabric. However, they are currently facing problems that largely depend on the succession process. This paper aims to expose the problems facing family businesses today and the elements to minimize them from the published literature on this topic.

Keywords: Family business; succession in family businesses; success of family businesses; problems.

\section{Resumo}

As empresas familiares estão no mundo, cada vez mais importantes, tanto econômicas como socialmente, porque em vários setores se tornaram motoristas do tecido comercial. No entanto, atualmente enfrentam problemas que dependem em grande parte do processo de sucessão. Este artigo pretende expor os problemas enfrentados pelas empresas familiares hoje e os elementos para minimizá-las da literatura publicada sobre esse tema.

Palavras chave: Negócio familiar; sucessão em empresas familiares; sucesso das empresas familiares; problemas.

\section{Introducción}

Las empresas familiares son en el mundo, cada vez más importantes, tanto a nivel económico como social, pues en diversos sectores se han convertido en motores del tejido empresarial.

Un importante número de emprendimientos en diversas partes del mundo inician bajo la forma de una empresa de familia, es decir, que las empresas son propiedad de una familia y son 
controladas por ésta. En este caso, la unidad básica de cohesión social llamada familia, sirve también como unidad básica para la organización económica. La empresa de familia en los hogares campesinos fue un hecho omnipresente, tanto en las sociedades agrícolas preindustriales como en las modernas, y formó la columna vertebral de la primera Revolución Industrial, tanto en Inglaterra como en Estados Unidos. En economías maduras, las nuevas empresas también comienzan en general como empresas de familia, que con el tiempo van adoptando diversas formas en la estructura de propiedad, algunas se desplazan a formas impersonales, mientras otras mantienen el componente familiar en su propiedad y control. (Macías R., 2011).

La empresa familiar es una organización en la que independientemente del tamaño y de las acciones que se realizan, la mayoría de la propiedad pertenece a una o más familias. Las empresas familiares son creadas por una o varias personas dotadas de una alta capacidad de esfuerzo, vocación, intuición y visión para desarrollar e implementar el proyecto empresarial al que destinan toda su energía y dedicación. (Pérez Molina A.I., 2012).

Este trabajo tiene como objetivo exponer los problemas que enfrentan hoy las empresas familiares y los factores de éxito a partir de la literatura publicada sobre este tema.

\section{Desarrollo}

Según Barbeito Roibal (s/f) las empresas familiares se enfrentan, independientemente de su localización geográfica, a los siguientes problemas propios de este tipo de organización:

- Problemas derivados del funcionamiento de la empresa familiar: debidos fundamentalmente a la confusión entre los flujos empresariales y los intereses familiares.

En cuanto a este problema Pérez Molina (2012), expone "Estos derivan de unir la propiedad y gestión y de combinar los objetivos empresariales y las metas familiares".

- Problemas de la sucesión en los que se encuentran:

- Los problemas fiscales, que se derivan del pago del Impuesto sobre Patrimonio y del Impuesto sobre Sucesiones y Donaciones, a los que tienen que hacer frente las empresas familiares cuando llega el momento del traspaso generacional. 
- Y los conflictos familiares derivados del traspaso generacional, debidos fundamentalmente a que el predecesor no estableció un plan de sucesión definido. (Barbeito Roibal S., s/f).

Así, Pérez Molina (2012) plantea diversos problemas derivados del funcionamiento de la empresa familiar: (Pérez Molina A.I., 2012).

- No crecimiento de la empresa familiar: Es habitual que en las familias empresarias crezcan en mayor proporción que la empresa familiar. Esto da lugar a que la organización no pueda cubrir todas las necesidades de la familia. A esto se suma que en ocasiones existe un exceso en la remuneración a ciertos miembros de la familia.

- Desmotivación del personal: Los miembros de la familia se desmotivan cuando no pueden acceder a información financiera de la empresa. Los trabajadores no familiares se desmotivan con facilidad por el trato preferente a los familiares (diferencias en la remuneración, poca libertad, menos opciones para la autorrealización personal, etc.).

- Incorporación a la empresa familiar: En las empresas familiares existen numerosos problemas cuando algún miembro de la familia, familiares políticos o terceros desean incorporarse en la empresa. No suelen existir normas sobre: quienes pueden entrar en la empresa, que preparación se requiere, como se determina el cargo y la responsabilidad, que hacer en caso de que el familiar no se adapte a la empresa, etc.

- Armonía familiar: En las empresas familiares es muy habitual romper la armonía familiar debido a conflictos entre generaciones, entre hermanos, entre familiares políticos, etc. Las disputas entre padres e hijos ocasionan importantes problemas y suelen ser debidas a la fuerte autoridad y desconfianza del padre y la intención del hijo de gestionar de manera independiente la empresa. Los desacuerdos entre hermanos ocurren, sobretodo, por la competencia, los celos y la subordinación entre ellos. También son habituales tensiones entre miembros ajenos y miembros de la familia. Esto es debido a que en las empresas familiares los cargos con poder suelen pertenecer a familiares, lo cual incomoda a profesionales externos, ya que estos no pueden realizar su trabajo adecuadamente. En el caso de que exista 
algún socio no familiar suelen existir desacuerdos en la destinación de las ganancias, en las políticas de remuneración y ascensos y en los planes futuros.

- Comunicación inadecuada: En las empresas familiares la información corre en todas las direcciones posibles, aparecen chismes, rumores, se manipula la información, etc. y por tanto se generan problemas entre los miembros de la organización.

- Remuneración: Surgen problemas derivados de la evaluación y la remuneración de los miembros de la familia.

Macías (2011) expone que los factores administrativos son la principal causa de desaparición de las empresas familiares, seguida por las cuestiones de orden financiero y político-fiscal. Así plantea que en lo administrativo:

1. Incide la carencia de un sistema administrativo, ya que la mayoría de las Empresas Familiares dirigen sus negocios en forma improvisada, sin reglas claras en los roles para manejar su negocio y tomar las decisiones importantes; son algunas de las fallas más comunes en estas empresas.

2. Determina el fracaso operar con un sistema inadecuado, ya que las empresas familiares deben tener una dirección especial estructurada en una cadena de mando con líneas de autoridad definidas, porque no puede mandar cualquiera, con estatutos que definan roles, responsabilidades y derechos de cada familiar- socio.

3. Manejar ineficientemente los recursos, pues en cierto sentido siempre habrá una disputa por los beneficios obtenidos de la actividad de la empresa para favorecer al sistema familiar y dejar sin recursos a la empresa; esta misma cercanía entre los miembros puede llegar a debilitar la organización y hará perder poder a la gerencia ya que es muy probable que los intereses particulares puedan filtrarse más fácilmente dentro de las metas y objetivos de la firma; por tanto, no se está cumpliendo cabalmente con los fines que persigue la organización como tal.

4. Carecer de control en el sentido de la delegación, la cual es una herramienta básica de la administración pues si ésta no se ejerce de manera eficaz puede llevar a las organizaciones al caos; en la empresa familiar este factor es uno de los más carentes ya que existen problemas 
para delegar y buscar una persona idónea en la cual puedan confiar las decisiones más importantes de la empresa.

5. Las grandes dificultades que enfrentan las Empresas Familiares para lograr la continuidad en los caminos generacionales; aproximadamente una de cada tres empresas tiene éxito en la sucesión a la siguiente generación. (Macías R., 2011).

Caroz(s/f) habla de garantizar el éxito en los cambios generacionales. Las conclusiones de su estudio coinciden en la necesidad de una evolución en la forma de entender la dirección y el liderazgo en las empresas familiares hacia la denominada dirección por valores, y son:

a. Es conveniente que los propietarios y los directivos de negocios familiares incidan en la potenciación y consecución de una visión orientada al largo plazo durante la etapa de propietario-controlador para que la empresa familiar pueda evolucionar satisfactoriamente hacia la sociedad de hermanos.

"Cabe destacar que la visión a largo plazo es fundamental para la continuidad de la empresa. Así mismo, es esencial elegir una estructura de propiedad para la próxima generación y establecer unas bases para la sucesión y la futura gestión de la empresa. Otro factor que el propietario debe tener en cuenta es evaluar el atractivo de la empresa e implementar una estrategia que motive a los sucesores a tener interés por el negocio" (Pérez Molina A.I., 2012).

"(...) en la empresa de familia, dependiendo de cada momento histórico, se produce una atomización de la propiedad, la cual recaerá en una u otra persona o en grupos de personas, en este caso vinculados por relaciones de consanguinidad. El propietario (fundador), que es a su vez dirigente de la compañía, es el único supervisor y quien lleva toda la carga del negocio; pero con el paso de los años dicho fundador debe ceder el poder a sus descendientes, ocurriendo en muchos casos un retraso en la sucesión que viene determinado por el no saber estructurar el problema ni por parte del fundador ni por el resto de la organización, de tal forma que la resistencia o negación a su retiro es más cómodo que tomar una postura activa para solucionarlo. Si llegado el momento de la sucesión el predecesor no acepta su retiro, puede llevar al estancamiento, desmotivación de los directivos y además a que la empresa no se adapte a los cambios que se suceden con el tiempo, de manera que ésta puede llegar a desaparecer" (Macías R., 2011). 
"La sucesión es una de las etapas más difíciles por las que debe atravesar una organización, porque no solo afecta a quien o quienes se les transfieren el mando y la propiedad, sino que también requiere de un plan adecuado de retiro para el fundador o fundadores, acorde con la importancia que han tenido en la Compañía" (Lacouture, 2005, p. 14).

b. En una sociedad de hermanos es necesario y oportuno lograr el máximo nivel posible de compromiso familiar y no familiar, a través de las derramas que aquél puede generar sobre éste, como factor clave para asegurar el éxito en la transición a la etapa de consorcio de primos.

"Otra consideración importante en una empresa es la profesionalización, es decir, es necesario definir la función de los propietarios y de los empleados, atraer y mantener a profesionales, etc." (Pérez Molina A.I., 2012).

c. En un consorcio de primos, los directivos y propietarios han de concentrar sus esfuerzos en otro valor, la armonía. (Caroz, s/f).

"Además hay que tener en cuenta que los miembros de la familia sólo se incorporen si están claramente capacitados para desempeñar responsabilidades de dirección y que usan sistemas de dirección profesionales. Otro factor esencial es avanzar en el diseño y en aplicación de sistemas que garanticen la liquidez a los propietarios" (Pérez Molina A.I., 2012).

La sucesión en la empresa familiar, que está llena de emociones indiscutiblemente, es sin lugar a dudas, un elemento clave para la continuidad del proyecto empresarial. En este caso ese proceso sucesorio debe prepararse mediante un plan de sucesión.

Se entiende por sucesión el relevo que protagoniza la siguiente generación en el proceso de sustitución de la generación que la antecede.

"Al margen de cuantos análisis quieran hacerse, lo primero que ha de acontecer en una empresa familiar para garantizar el éxito de la sucesión es que tanto el predecesor como el sucesor estén alineados en sus objetivos y voluntades: el primero debe estar dispuesto a ceder el control y estar convencido de que el sucesor está preparado para asumir el reto; y por el otro lado, el sucesor debe estar capacitado para tomar el relevo y, además, querer hacerlo" (Familiar, 2007). 
Elementos importantes para el éxito en la sucesión en las empresas familiares es la preparación del sucesor, la preparación de la organización, la armonización de las relaciones empresa/familia y la preparación para la época de la jubilación del predecesor.

"Las empresas familiares se enfrentan cada vez más a situaciones complejas que afectan no sólo el destino de ésta, sino también el del familiar que se encuentra en proceso de retiro. La salida de este último tiene un impacto tanto para su familia, como para los empleados y aquellos terceros que tengan alguna relación de negocios. En el caso de algunas empresas familiares cuando el familiar que se retira, se ha convertido en la base de generación de riqueza de todo un grupo familiar y éste es el que provee los fondos que alimentan y visten a dos o tres generaciones al mismo tiempo, la trascendencia del suceso suele ser significativa. Adicionalmente, dicho evento puede tener mayor relevancia cuando la empresa familiar representa un motivo de orgullo para los miembros de la familia y conlleva a un apego emocional profundo. Sin embargo, la sucesión no debe verse como un motivo de desajustes económicos y emocionales, sino como un proceso natural" (Galaz Y., 2010).

Es por todo ello que todo empresario familiar debe planear el proceso de sucesión, como normal y exitoso.

A continuación presentamos algunos factores que pudieran ocasionar resistencia a la sucesión: (Galaz Y., 2010)

1. Falta de información: La inexistencia de información para la planeación, el diseño y la implantación de un plan de sucesión es uno de los principales factores que provocan resistencia. Por ello, contar con información es imprescindible para el adecuado manejo de ciertas situaciones familiares, además de ser un arma de conocimiento que elimina ambigüedades y confusiones dentro de las familias.

2. Factores culturales: Puede existir rechazo a la sucesión debido a factores relacionados con la propia idiosincrasia, a la educación recibida y a la influencia de generaciones predecesoras.

3. Amenaza al estatus quo: Esta sensación se debe a la resistencia a modificar la estructura organizacional en comparación a los esquemas, valores, costumbres y normas arraigados en la empresa. Asimismo este cambio podría representar a la vista del fundador una amenaza plena al 
estatus quo conquistado a lo largo de su vida empresarial, es decir, cuanto más haya invertido una persona en el sistema de operación del negocio, mayor será la resistencia a aceptar un cambio.

4. Retención de poder: Toda organización está expuesta a cambios y en consecuencia existe la posibilidad, de que aquellos individuos o grupos que vean amenazada su posición en la organización manifiesten resistencia a los mismos.

5. Clima organizacional: Previo a iniciar el proceso de sucesión se deberán identificar aquellos factores negativos que afecten el clima organizacional, cuanto más negativo sea éste, más complejo será conducir a la organización hacia el modelo de sucesión planteado.

6. Miedo al fracaso: La incertidumbre que puede generarse en el miembro de la familia que se retira respecto a que tan viable puede resultar la sucesión y si es el momento idóneo para llevar a cabo el proceso, se encuentra latente debido al temor de no estar seguro de que el o los sucesores se encuentren preparados para tomar el control de la empresa familiar.

Ahora bien, existen algunas medidas para disminuir la resistencia al cambio tras la implementación del plan de sucesión y son: (Familiar, 2007)

1. Identificar aquellos actores que se resisten al cambio y llevar a cabo un diagnóstico profundo sobre las causas que propician dicha reacción.

2. Fomentar e impulsar el cambio de manera transparente, informando del proceso y plan de sucesión a los diferentes implicados.

3. Involucrar a todos los actores en la implementación del plan de sucesión como coparticipes del mismo.

4. Sucesión familiar: Herramienta para preservar el patrimonio Si bien no es posible contar con cifras comparativas exactas a nivel internacional, existen tendencias que indican que las empresas familiares tienen una vida corta.

\section{Conclusiones}

Los problemas de las empresas familiares surgen, como se ha visto, por diversos motivos. Si estos no se tratan de manera adecuada, la supervivencia de la empresa está en peligro. 
Existen herramientas que viabilizan el éxito de la empresa familiar, como son la profesionalidad y formación de sus miembros, y la planificación y ejecución correcta de la sucesión generacional. Esta última cuestión es una de las causas que más prevalecen en el fracaso de las empresas familiares.

A diferencia de otros modelos empresariales, en los que la continuidad y transcendencia de los mismos depende de la competitividad en mayor parte, en la empresa familiar es necesario, además de la competitividad, establecer un plan de sucesión de la empresa con la finalidad de hacer que la empresa perdure bajo la administración y directrices familiares.

La decisión de no hacer nada hasta el fallecimiento del fundador, es errada; pues esto lleva un proceso de preparación y liderazgo del sucesor, que en la literatura, de manera conclusiva, se concentra en la conformación del consejo familiar, la realización del plan de sucesión y las políticas para la incorporación de familiares a la compañía, la compartición de las expectativas de futuro, la solución de conflictos familiares, la evaluación de los empleados familiares, la implementación de planes y programas familiares para la perpetuidad de la empresa, la transmisión de los valores, el desarrollo del liderazgo y la interacción entre la familia y la empresa.

\section{Referencias bibliográficas}

Ávila Forero JS. (2016). Diseño de material didáctico para la enseñanza de anatomía. IFDP`16 Systems \& Design:Beyond Processes and Thinking.

Barbeito Roibal S., S. E. (s/f). Visión europea del proceso de sucesión en la empresa familiar.

Caroz. (s/f). La respuesta cultural a los problemas evolutivos de la empresa familiar.

Familiar, C. d. (2007). El proceso de sucesión en la empresa familiar. Expansión.

Galaz Y., R. S. (2010). Sucesión en la Empresa Familiar. Boletín Gobierno Corporativo.

Gal-Iglesias B., B.-B. I.-A. (2009). Nuevas metodologías docentes aplicadas al estudio de la fisiología y la anatomía: estudio comparativo con el método tradicional. EDUC MED 12 (2). 
García Taya J.A., A. R. (2014). El uso de la tecnología en la enseñanza de la anatomía en México y su comparación con la enseñanza internacional. Revista de la Facultad de Medicina de la UNAM 3232.

Macías R., V. H. (2011). Sucesión en empresas familiares. Análisis desde la teoría de la agencia. Caso: Caldas. Criterio Libre 9 (15).

Mompeó-Corredera B. (2014). Metodologías y materiales para el aprendizaje de la anatomía humana. Percepciones de los estudiantes de medicina 'nativos digitales'. FEM vol.17 no2.

Montero Cofre C. I. (s/f). El propósito de las TIC en el estudio de la anatomía humana. Aplicación de Blended Learning en la asignatura de Anatomía Humana para estudiantes de primer año de la carrera de Medicina. VIII Jornadas Internacionales de Innovación Universitaria Retos y oportunidades del desarrollo de los nuevos títulos en educación superior.

Pérez Molina A.I., S. V. (2012). La continuidad en la empresa familiar. Análisis de casos. 3Ciencias Revista de investigación.

Raúl. (s/f). Modelos anatómicos interactivos para la clase. Obtenido de http://www.noticiasusodidactico.com/blog/author/raul/

Sánchez A. (2017). Anatomy Lab, una manera increíble de explorar la anatomía y al mismo tiempo mejorar el aprendizaje dentro de las universidades. Obtenido de https://www.azulweb.net/ciencia/

Sánchez Hilda L., S. L. (s/f). Una propuesta de aula extendida para mejorar el rendimiento académico de los alumnos del curso de anatomía i a través del uso de las TIC. IV Congreso Nacional y III Congreso Internacional de Enseñanza de las Ciencias Agropecuarias 824.

Santana A. (2016). ¿Cómo enseñar Anatomía Humana en el siglo XXI? Obtenido de http://www.udla.cl/cienciasdelasalud

Shutterstock. (2017). 5 herramientas tecnológicas para mejorar tus clases. Obtenido de http://noticias.universia.es/educacion 